\title{
Viscosity and Morphology Modification of Length Sorted Single-Walled Carbon Nanotubes in PIB Matrices
}

\author{
Hanxiao Huang, ${ }^{1}$ Bohao Li, ${ }^{1}$ Clayton E. Simien, ${ }^{2}$ and Daneesh O. Simien ${ }^{1}$ \\ ${ }^{1}$ Department of Materials Science and Engineering, Engineering School, University of Alabama at Birmingham, \\ Birmingham, AL, USA \\ ${ }^{2}$ Department of Physics, College of Arts and Sciences, University of Alabama at Birmingham, Birmingham, AL, USA
}

Correspondence should be addressed to Daneesh O. Simien; dsimien@uab.edu

Received 28 April 2017; Revised 12 July 2017; Accepted 14 August 2017; Published 18 September 2017

Academic Editor: Xiaowei He

Copyright (C) 2017 Hanxiao Huang et al. This is an open access article distributed under the Creative Commons Attribution License, which permits unrestricted use, distribution, and reproduction in any medium, provided the original work is properly cited.

\begin{abstract}
This work evaluates the effectiveness of nanoscale particulates in producing non-Einstein-like responses in polymer matrices, to reduce their negative effects in low shear rate processing. This is of value to material processing applications which encompass extrusion, flow into cold mold, and generalized processing of nanocomposites. Through control and understanding of the structure processing relationships entailed through nanoscale additive materials, we begin to manage dispersion characteristics for more reliable and defect-free product development. In pursuit of identifying system characteristics that produce non-Einstein-like responses we isolate and characterize homogenous fractions of single-walled carbon nanotubes (SWNTs) with singular lengths. This enables the definition of a well-defined nanoscale particulate phase, within the polymer matrices. The effect of nanotube length and weight fraction on the polyisobutylene (PIB) matrices was evaluated with thermal and rheological testing. Our findings show that the viscosity of the produced nanocomposite systems has a length dependence and does not demonstrate the expected monotonous increases in the viscosity with an increase in weight fraction of nanotube additive within the matrix, demonstrating a non-Einstein-like viscosity response. Furthermore, we demonstrate length dependent crystallization in the studied systems, as an intermediate length nanotube initiates crystallization of polyisobutylene (PIB) affecting viscosity and mechanical properties.
\end{abstract}

\section{Introduction}

Single-walled carbon nanotubes (SWNTs) are an example of the more recently discovered nanoscale materials with unique structure and properties. Composed of single carbon atoms in a rod-like cylindrical pattern, it has a high degree of structural perfection [1]. Furthermore, the dominating covalent bonded structure provides extremely high modulus and strength, even comparable to that of steel, but only at a sixth of its density. Advantages in mechanical strength and thermal and electrical conductivity make carbon nanotubes ideal additives in polymer nanocomposites [2-4]. However, there are several processing concerns in generating nanoscale composites, such as the dispersion of the nanoscale phase within the polymer matrix and the initiation of large increases in viscosity with the addition of nanoscale additives and poor polymer-nanotube interface, all of which ultimately contribute to the formation of defects which do not facilitate the expected strengthening in mechanical or electrical properties which should be achieved.

In processing SWNT-polymer nanocomposites, viscosity increase can be a primary challenge. It affects flow rates in different temperature zones at different shear rates. It may affect the flow rates and nanoparticle distributions achieved in printing nanoscale inks or defect formation in flow into cold molds for nanocomposite product development. In general, Einstein predicts that the viscosity of Brownian particles is solely a function of its volume in the suspending liquid [5]. Therefore the inclusion of a particle in a suspending phase should increase the viscosity of the system. However it has been shown that nanoscale inclusions may demonstrate non-Einstein-like behavior where a decrease or no change in the viscosity of overall system is observed. This atypical type of system behavior occurs when the nanoparticles provide confinement and surface effects and cause conformational changes to the macromolecules of the polymer system 
present. Uniformly dispersed nanoparticles reduce the entanglement of polymer chains, while the blend viscosity reduces with the free volume associated with the nanoscale filler or particles, due to their rigid and stiff characteristics rendering non-Einstein-like behavior. The prerequisite of this behavior is that the nanoscale filler must have comparable scale to the radius of gyration of polymer, at least, in one dimension, for example, length, width, or diameter. The radius of gyration, $R_{g}$, is defined as the distance of a collection of atoms from their common center of gravity and influenced by the molecular weight, stiffness, and viscosity of the polymer [5-7].

In previous studies by Mackay et al., non-Einsteinlike behavior is demonstrated where spherical nanoparticles (radius of $3 \mathrm{~nm}-5 \mathrm{~nm}$ ) synthesized by crosslinking polystyrene (PS) were added to a polystyrene matrix which had $R_{g}$ of $7.5-15 \mathrm{~nm}$. These systems demonstrated the atypical non-Einstein-like responses which can be observed in nanoparticulate systems. In these systems the spherical particles increased the free volume of the system and also reduced the entanglement of the matrix. The composite displayed a reduction in the low shear rate viscosity with addition of up to $10 \mathrm{wt} . \%$ nanoparticle, later showing the expected increases in viscosity at higher loadings associated with the increased stiffness attributed to the present nanoparticles [5]. Jain et al. [8] studied the viscosity reduction from silica particles in polypropylene (PP) matrices, in which they attributed the atypical viscosity responses to the selective physisorption of long macromolecular chains onto the surface of silica particles due to similar surface chemical characteristics. In addition, Xie et al. [9] found a viscosity decrease in $\mathrm{PVC} / \mathrm{CaCO}_{3}$ systems due to a ball bearing effect, while Cho and Paul [10] attributed viscosity reduction in their systems to the molecular degradation and slip at wall for nylon-clay systems. In each of these works it is observed that atypical viscosity can be achieved depending on the particulate morphology, treatment, or interaction dynamics. In our systems, we observe effects that may be more narrowed to interactions such as steric hindrance and a reduction of polymer entanglement. In this process, uniformly dispersed nanoparticles reduce the entanglement of polymer chains while the blend viscosity reduces with the free volume associated with the nanoscale filler or particles, due to the rigid and stiff characteristics of the length sorted single-walled carbon nanotubes (SWNTs) rendering non-Einstein-like behavior. Roberts et al. [11] found that clusters of silica caused viscosity reduction to the linear poly(dimethylsiloxane) when the nanoparticles were less than $0.7 \mathrm{~nm}$ in size, which facilitated plasticizing effects. Tuteja et al. used PS nanoparticles and studied its viscosity reduction effect, at different molecular weights of the linear PS matrix [12]. They concluded that, to induce non-Einstein-like viscosity behavior, the polymer must be entangled, and the separation distance between nanoparticles should be comparable to the polymer radius of gyration. They also found that fullerenes resulted in a similar viscosity reduction at a mass fraction of 0.02 [13]. Furthermore, a more quantitative study was conducted by Schmidt et al. [14], in which they defined a critical nanoparticle-to-polymer $R_{g}$ ratio to induce the transition from plasticization (reduced viscosity) to reinforcement (increased viscosity). In their work blending polysilicate with poly(dimethylsiloxane), the critical ratio was found to be 0.18 , at $298 \mathrm{~K}$ and a nanoparticle weight fraction of 0.17 . Further research also demonstrates that carbon nanotubes can result in steric hindrance and viscosity reduction. Petriccione et al. found that the viscosity of bifunctional epoxy-ammine systems showed a reduction when the weight fraction of multiwalled carbon nanotubes increased from 0.096 to 0.190 wt.\% [15]. Similarly, Zhang et al. found that, at $0.1-0.2 \mathrm{wt}$.\% fraction, SWNTs resulted in a significant viscosity reduction in polyethylene (PE) systems, which then showed viscosity increases again at $1 \mathrm{wt} . \%$ due to the formation of nanotube networks [16].

In this publication, we explore non-Einstein-like viscosity behavior in single-walled carbon nanotube (SWNT) composite systems by controlling the weight fraction and the length and diameter of nanotube fractions used as additives to PIB matrices. Our goal is to ensure that these SWNT parameters are comparable to or smaller than the radius of gyration of the PIB molecules in the matrices, with the goal of optimizing our understanding of viscosity control in these nanoscale systems. In evaluating our SWNT-polymer nanocomposite systems, we also find evidence of nanotube induced polymer crystallization. It is well known that nanotubes are one of the most effective nucleation agents for the heterogeneous crystallization of polymers, particularly for crystalline and semicrystalline polymers [17-20]. Li et al. demonstrated, for example, that selected polyethylene (PE) oligomers at a low molecular weight, $800 \mathrm{~g} / \mathrm{mol}$, demonstrated evidence of crystallization at nanotube sites [17].

In our work, the as-received single-walled carbon nanotubes (SWNTs) were separated by length using density gradient ultracentrifugation [21]. Polyisobutylene (PIB) was selected as the incorporating polymer matrix, and homogenous fractions of SWNTs of varying lengths and weight fractions were isolated and blended with the PIB. The effects of, both, nanotube length and weight fraction were studied on the viscosity, thermal behavior, and crystallization of the PIB matrix. We believe this study will broaden the understanding of the fabrication and control of SWNTpolymer nanocomposites and enhance our understanding of nanoparticle usage as additives for thermal applications such as in extending the life of engine oils, for which PIB is sometimes used as a base oil.

\section{Results}

2.1. UV-Vis-NIR Spectroscopy. Ultraviolet-visible-near infrared (UV-Vis-NIR) spectroscopy was first conducted on the as-received nanotubes which were presorted via ultracentrifugation to remove excess carbon and catalytic materials, shown in Figure 1. The chiralities $(n, m)$ of the presorted nanotube solution were identified. Subsequently, further separation to isolate homogenous length fractions of SWNTs by layers in a density gradient was performed. A UV-Vis-NIR analysis was preformed and the spectra were analyzed for each length separated layer retrieved after the separation process. A normalization was applied at $775 \mathrm{~nm}$, where no $\pi$-plasmon transitions are located, to identify variations which occur in the comparative intensity of 


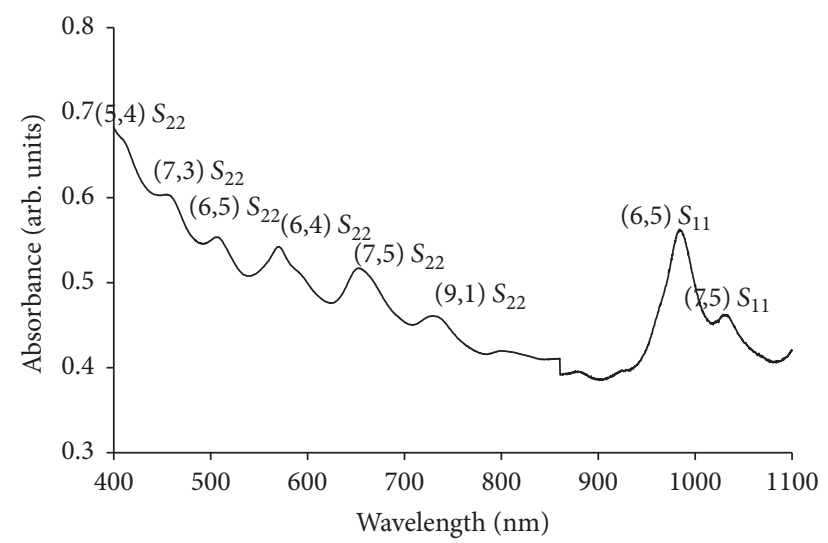

FIGURE 1: UV-Vis spectroscopy curve of presorted nanotubes sample.

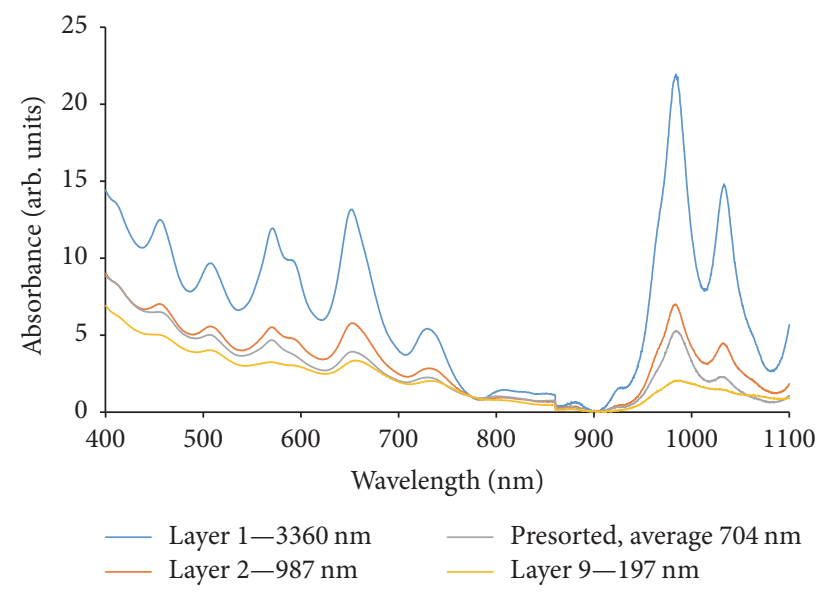

FIGURE 2: Normalized UV-Vis spectroscopy curves of three selected length sorted SWNT samples.

homogeneous length fractions; the result is shown in Figure 2. The length of the SWNTs was estimated by the equation below: $L(\mathrm{~nm})=\left(A_{984 \mathrm{~nm}} / A_{775 \mathrm{~nm}}-1\right) * 160.4 \mathrm{~nm}$ [21]. The normalized peak ratio and calculated nanotube lengths are summarized in Table 1. According to Table 1, the length of nanotube decreases as depth of separation layer increases.

2.2. Scanning Electron Microscopy. After length separation, nanotube solutions of two lengths $3360 \mathrm{~nm}$ and $987 \mathrm{~nm}$ were selected for study. The presorted nanotubes of mixed lengths (with an average length parameter of $704 \mathrm{~nm}$ ) were selected as the control group. According to Figures 3(a) and 3(b), a considerable length difference occurs and can be isolated after sorting. This length difference is consistent with calculations from the UV-Vis-NIR analysis.

2.3. Differential Scanning Calorimetry. The glass transition temperatures $\left(T_{g}\right)$ of all samples were recorded using Differential Scanning Calorimetry (DSC). As shown in Table 2, the pristine PIB has $T_{g}$ at $-69.59^{\circ} \mathrm{C}$. As the weight fraction of the nanotubes present in the composite increases to 0.02 and $0.05 \mathrm{wt} . \%, \mathrm{~T}_{g}$ is reduced by approximately $7.35^{\circ} \mathrm{C}$ and $5.82^{\circ} \mathrm{C}$, respectively, whereas above $0.1 \mathrm{wt} . \%$ much smaller changes in the $T_{g}$ are observed, which is an atypical response. The glass transition of a polymer will generally be affected by its environment when the chain length is within several nanometers of the secondary phase [22]. The larger changes in $T_{g}$ experienced by the addition of less than 0.08 wt. $\%$ nanoparticle may be attributed to the nanoparticles being near their percolation threshold and the initial onset of an interconnected nanotube network, which generated a "nanoscale-effect" which is isolated to that critical concentration regime [23].

The heat flow curves of all length sorted nanotubes at three weight fractions are shown in Figures 4, 5, and 6. Among all the lengths, nanotubes with lengths 638, 384, and $197 \mathrm{~nm}$ result in observable melting peaks and the area of the peaks was summarized in Table 3. According to Table 3, $638 \mathrm{~nm}$ length samples display the highest peak area indicating the highest crystallinity degree. In contrast, 384 and $197 \mathrm{~nm}$ samples display a reduced crystallinity degree while the 3360 and $987 \mathrm{~nm}$ samples remained highly amorphous. As weight fraction of nanotube increases, the crystallinity degree decreased among all lengths evaluated.

In observing the morphology of the SWNT-PIB composites, there is a dramatic difference in the samples depending on the length of the incorporated SWNT phase. The presorted samples avg., length $704 \mathrm{~nm}, 987 \mathrm{~nm}$, and $3360 \mathrm{~nm}$ demonstrate an amorphous "gel-like" structure, while the shorter length SWNT composites of $638 \mathrm{~nm}$ and below demonstrate a crystalline structure. The longer length SWNT samples are likely providing more steric hindrance and network structures, while the shorter length SWNTs are serving as nucleation sites for PIB crystallization.

2.4. Rheological Testing. Dynamic viscosities of presorted nanotubes-PIB mixtures, at multiple weight fractions, are shown in Figure 7, and the dynamic viscosity at frequency $0.1 \mathrm{rad} / \mathrm{s}$ was recorded in Table 4 as the final viscosity value for further discussion. According to Table 4, dynamic viscosity increases dramatically from 1 to $5 \mathrm{wt}$.\%. However, samples with nanotube weight fractions not higher than 0.05 wt. $\%$ have dynamic viscosity smaller than that of the virgin PIB. This $0.02 \mathrm{wt}$ \% sample represents a sample which is close to the critical percolation concentration of the nanotubes incorporated and is the first indicator of possible nonEinstein-like behavior among these nanocomposite systems.

The dynamic viscosity of nanocomposites made from select isolated fraction of homogeneous single length nanotubes was shown in Figures 8, 9, and 10. Also, the dynamic viscosity of each sample at $0.1 \mathrm{wt}$. $\%$ was recorded in Table 5 for further discussion. According to Table 5, the dynamic viscosity is the highest among intermediate length $638 \mathrm{~nm}$ and shorter lengths 384 and $197 \mathrm{~nm}$ nanotube composites. In contrast, the 3360 and $987 \mathrm{~nm}$ length SWNT samples have smaller dynamic viscosity while those of presorted nanotubes (mixed lengths with avg. length of $704 \mathrm{~nm}$ ) have the smallest value. Meanwhile, as nanotube weight fraction increases from 0.1 to 0.25 and further to $0.5 \mathrm{wt}$.\%, the dynamic viscosity of all 
TABLE 1: Normalized peak ratio and calculated length and concentration of nanotube.

\begin{tabular}{lcccc}
\hline $\begin{array}{l}\text { Layer after } \\
\text { length } \\
\text { separation }\end{array}$ & $\begin{array}{c}A_{984 \mathrm{~nm}} / A_{775 \mathrm{~nm}} \\
\text { after } \\
\text { normalization }\end{array}$ & $\begin{array}{c}\text { Calculated } \\
\text { length [nm] }\end{array}$ & $\begin{array}{c}A_{775 \mathrm{~nm} \text { before }} \\
\text { normalization }\end{array}$ & $\begin{array}{c}\text { Calculated } \\
\text { concentration } \\
{[\mathrm{mg} / \mathrm{ml}]}\end{array}$ \\
\hline 1st & 21.97 & 3360 & 0.587 & 0.411 \\
2nd & 14.98 & 2267 & 0.384 & 0.269 \\
3rd & 7.00 & 987 & 0.250 & 0.175 \\
4th & 4.82 & 638 & 0.100 & 0.070 \\
5th & 3.24 & 384 & 0.207 & 0.145 \\
6th & 2.58 & 279 & 0.182 & 0.127 \\
7th & 2.39 & 248 & 0.191 & 0.134 \\
8th & 2.23 & 223 & 0.248 & 0.174 \\
9th & 2.07 & 197 & 0.221 & 0.155 \\
Presorted & 5.23 & 704 & 0.424 & 0.297 \\
nanotube & & & & \\
\hline
\end{tabular}

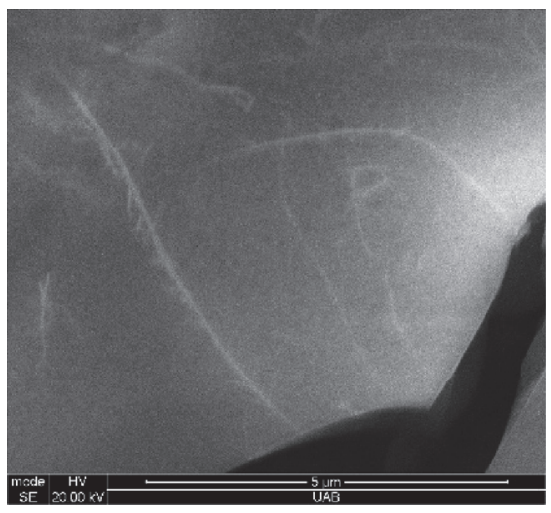

(a)

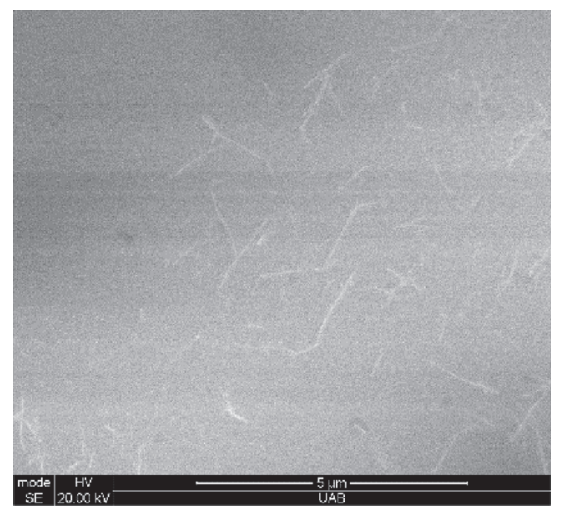

(b)

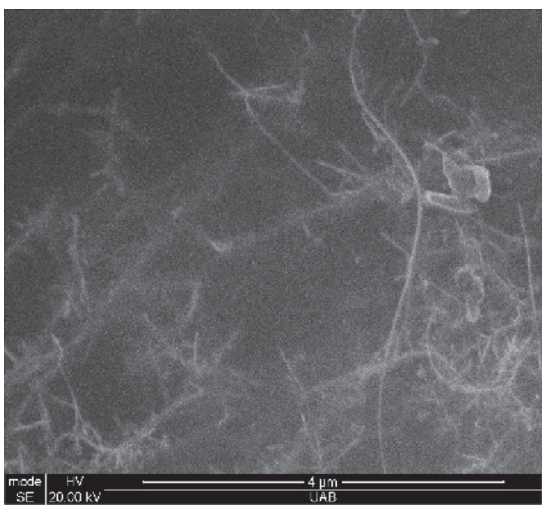

(c)

FIGURE 3: SEM images for SWNT fractions of lengths (a) $3360 \mathrm{~nm}$ and (b) $987 \mathrm{~nm}$ and (c) presorted sample (avg. $704 \mathrm{~nm}$ ).

TABLE 2: Effect of nanotube weight fraction on glass transition temperatures.

\begin{tabular}{lc}
\hline SWNT weight fraction [wt.\%] & $T_{g}\left[{ }^{\circ} \mathrm{C}\right]$ \\
\hline 0 & -69.59 \\
0.02 & -76.94 \\
0.05 & -75.41 \\
0.1 & -66.30 \\
0.25 & -65.45 \\
0.5 & -66.18 \\
1 & -65.42 \\
5 & -66.44 \\
\hline
\end{tabular}

samples increased, but more significantly in 3360 and $987 \mathrm{~nm}$ samples.

In isolating the length of the nanotube in singular fractions and exploring the resulting structure-property relationships, we see that very different properties can be obtained, for example, in the crystallization behavior of the matrix. The presorted samples having an average length of c.a. $704 \mathrm{~nm}$ but containing both long and short length nanotubes
TABLE 3: Melting peak area of SWNT/PIB at varying lengths and weight fractions.

\begin{tabular}{lccc}
\hline $\begin{array}{l}\text { Melting peak } \\
\text { area }\end{array}$ & $0.1 \mathrm{wt} . \%$ & $0.25 \mathrm{wt} . \%$ & $0.5 \mathrm{wt} . \%$ \\
\hline $638 \mathrm{~nm}$ & 111.60 & 75.87 & 67.08 \\
$384 \mathrm{~nm}$ & 85.21 & 46.79 & 21.01 \\
$197 \mathrm{~nm}$ & 66.58 & 34.40 & 17.53 \\
\hline
\end{tabular}

demonstrate such behavior. The longer length nanotubes, present in this starting sample, result in a retardation of crystallization behavior in the PIB matrix, which is shown in the data for the (mixed length) presorted nanotubes. This behavior is different from that which is observed when we evaluate a SWNT sample with homogeneous length of $638 \mathrm{~nm}$, for example, which demonstrates a different crystallization response.

\section{Discussion}

3.1. UV-Vis-NIR Spectroscopy. In order to generate isolated lengths species from the as-received fractions an ultracentrifuge technique is used on the nanotubes which have been 


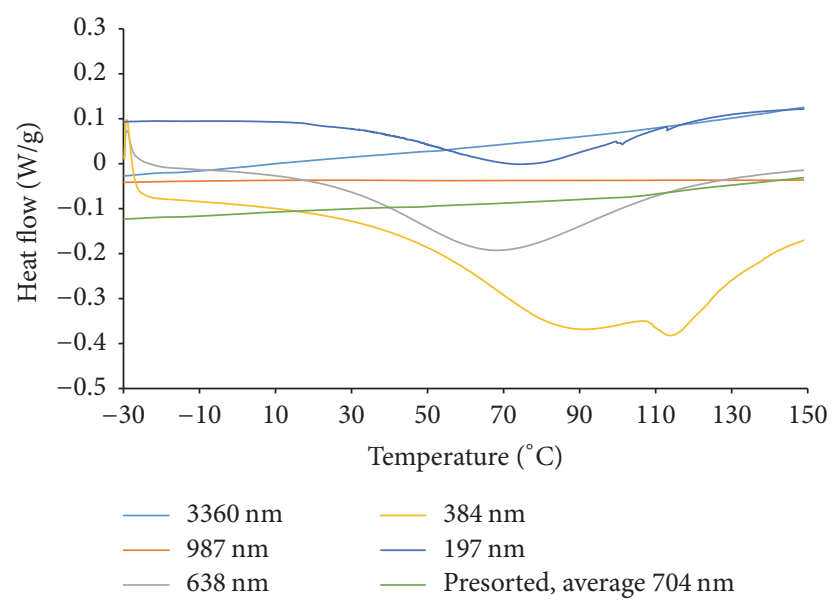

Figure 4: Heat flow of length sorted nanotube-PIB composites, 0.1 wt. $\%$.

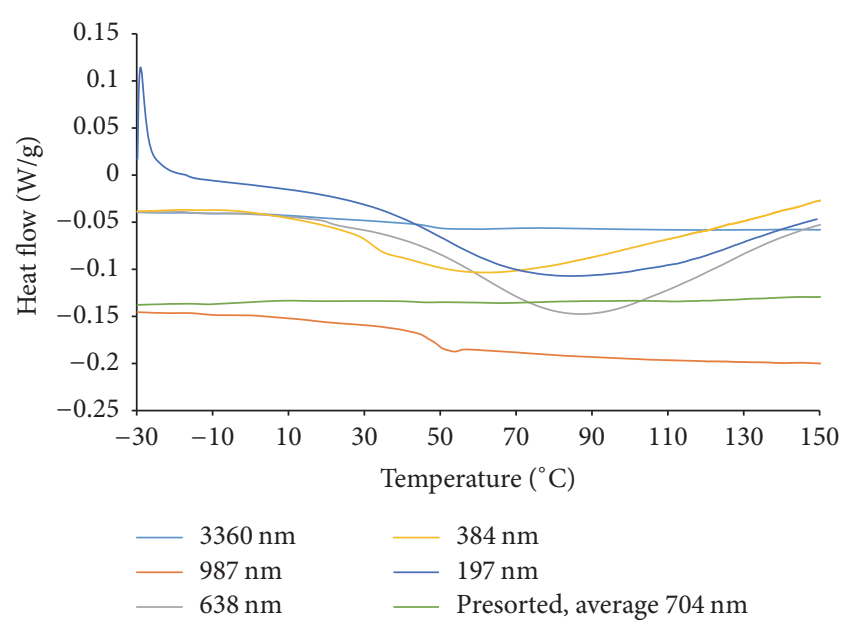

FIGURE 5: Heat flow of length sorted nanotube-PIB composites, 0.25 wt. \%.

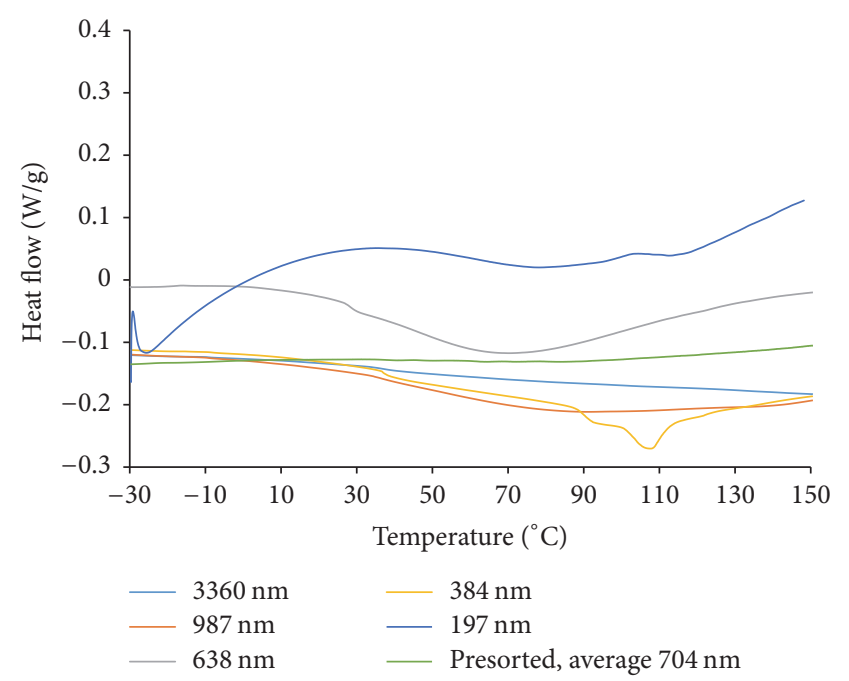

FIGURE 6: Heat flow of length sorted nanotube-PIB composites, 0.5 wt. $\%$.
TABLE 4: Viscosity value of presorted nanotube mixtures at various weight fractions.

\begin{tabular}{lc}
\hline $\begin{array}{l}\text { Presorted SWNT weight } \\
\text { fraction [wt.\%] }\end{array}$ & Viscosity [Pa*s] \\
\hline 0 & 23,290 \\
\hline 0.02 & 19,910 \\
0.05 & 20,510 \\
0.1 & 43,120 \\
0.25 & 59,860 \\
0.5 & 88,690 \\
1 & 104,800 \\
5 & 784,900 \\
\hline
\end{tabular}

TABLE 5: Viscosity values of length sorted nanotube mixtures at varying lengths and weight fractions at $0.1 \mathrm{rad} / \mathrm{s}$.

\begin{tabular}{lccc}
\hline Dynamic viscosity [Pa·s] & $0.1 \mathrm{wt} . \%$ & $0.25 \mathrm{wt} . \%$ & $0.5 \mathrm{wt} . \%$ \\
\hline $3360 \mathrm{~nm}$ & 171,500 & 575,200 & $1,124,000$ \\
$987 \mathrm{~nm}$ & 107,400 & 176,000 & 381,000 \\
$638 \mathrm{~nm}$ & 811,000 & $1,092,000$ & $1,639,000$ \\
$384 \mathrm{~nm}$ & 646,900 & 821,700 & $1,050,000$ \\
$197 \mathrm{~nm}$ & 492,100 & 522,800 & 589,000 \\
Presorted, mixed length, & 43,120 & 59,860 & 88,690 \\
average 704 nm & & 23,290 \\
\hline PIB
\end{tabular}

suspended in a density gradient fluid. This technique was initially proposed by Fagan et al. who successfully separated nanotubes by length [21]. In this process, the difference in the buoyancy and frictional force between nanotubes of varying lengths is exploited in order to achieve separation in a transient regime. According to Fagan et al. [21, 24], nanotubes with larger length have a lower frictional coefficient and tend to travel with less opposition to the centrifugal acceleration. Thus, longer length nanotube accumulates near the top of the centrifuge tube, when suspended in a density gradient fluid and subjected to an accelerating field, while the shorter ones tend to sediment deeper. Multiple lengths of nanotube were isolated using this method which will be used for further experiments including 3360, 987, 638, 384, and $197 \mathrm{~nm}$.

3.2. Scanning Electron Microscopy. According to Figures 3(a) and $3(\mathrm{~b})$, the observed length is consistent with calculation from UV-Vis-NIR analysis. As introduced above, the nanoscale filler has the potential to cause conformational change and modified rheological behavior of the polymer to which it is added. On one hand, the nanoscale filler reduces the entanglement of polymer. On the other hand, nanoscale particles are highly rigid and stiff. In other words, they are not deformable. Thus, they add free volume themselves. The perquisite for conformational change is that the nanoscale filler must have comparable size with the radius of gyration $\left(R_{g}\right)$ of polymer. More specifically, at least one dimension should be in the same order of size with $R_{g}$, including length and diameter. Fetters et al. [7] developed a calculation of 


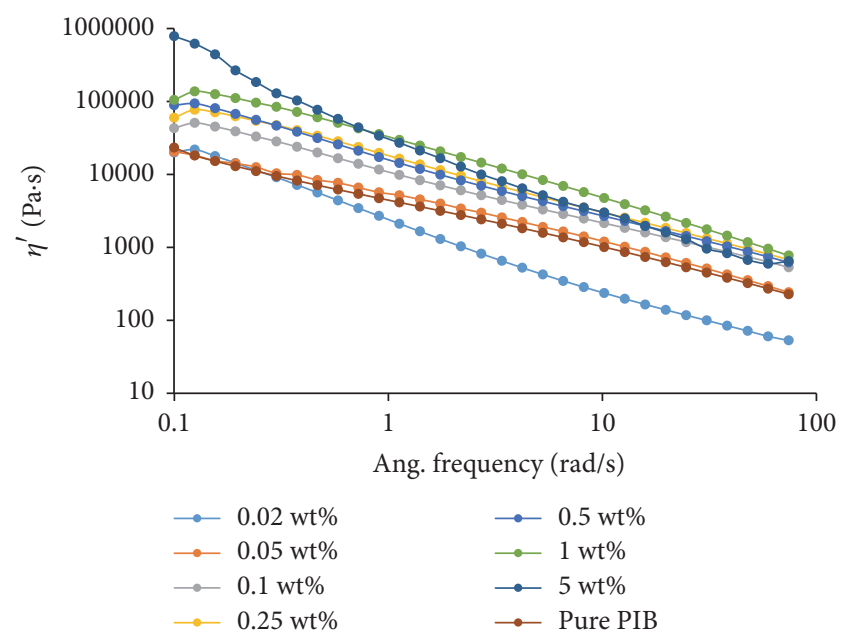

FIGURE 7: Viscosity curve of presorted nanotube mixtures (the same average length $704 \mathrm{~nm}$ ) at various weight fractions.

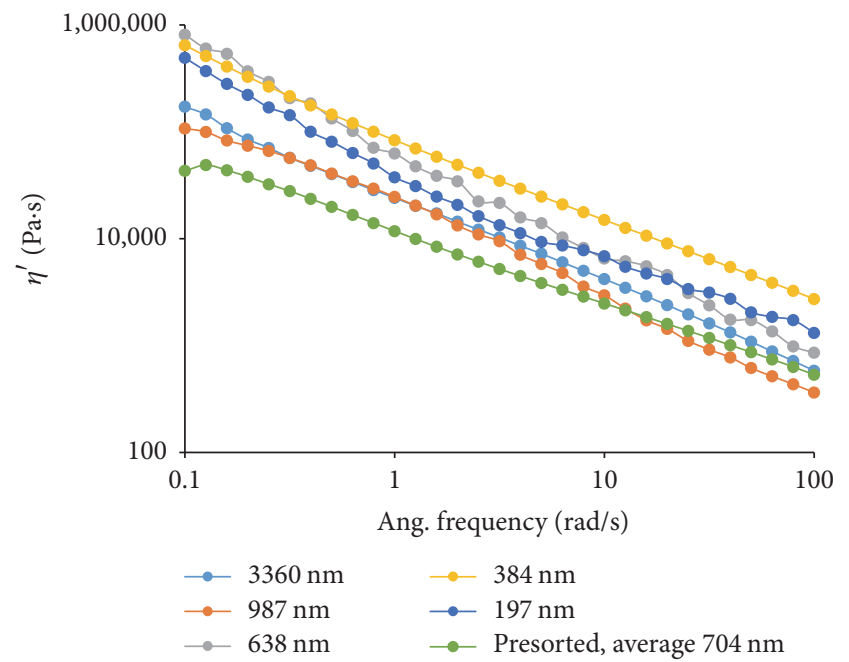

Figure 8: Dynamic viscosity of nanotube at various lengths (0.1 wt.\% loading of SWNT, at data point $0.1 \mathrm{rad} / \mathrm{s}$ ).

radius of gyration for various polymers including polybutadiene, polyisoprene, polystyrene, and polyisobutylene. Data for PIB was collected in theta solvents benzene and isoamyl isovalerate at room temperature. The extent of distortion of polymer chain can vary depending on its molecular weight range and solvent environment. Thus, various relationships are generated depending on these variables.

When the radius of gyration is collected in theta solvents isoamyl isovalerate, the applicable molecular weight range is $3.91 * 10^{5}<M_{n}<4.7 * 10^{6}$, and the radius of gyration is

$$
R_{g}=2.62 * 10^{-2} * M_{n}^{0.511}
$$

If a broader applicable range of molecular weight is required, cyclohexane is considered. In the molecular weight range

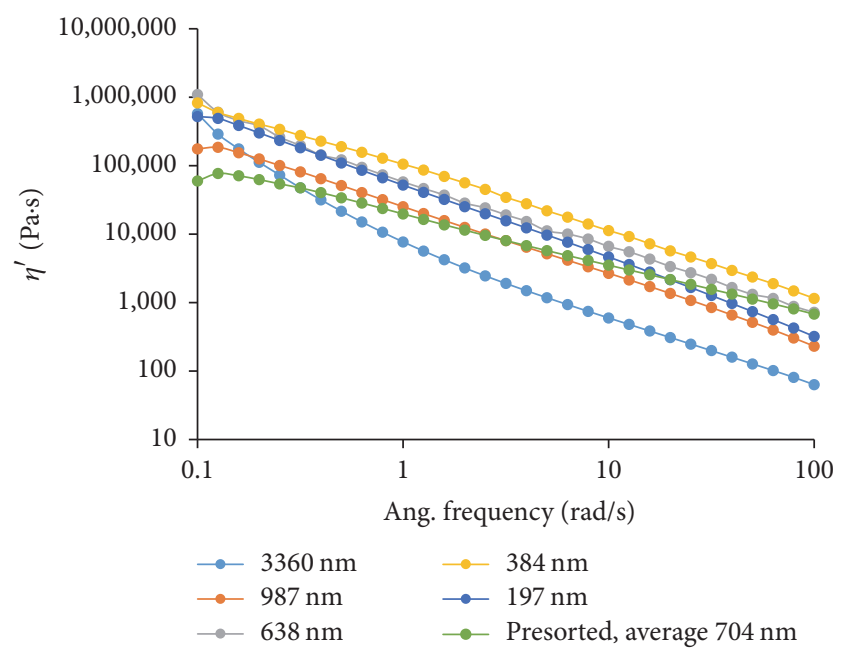

FIGURE 9: Dynamic viscosity of nanotube at various lengths ( $0.25 \mathrm{wt}$.\% loading of SWNT, at data point $0.1 \mathrm{rad} / \mathrm{s}$ ).

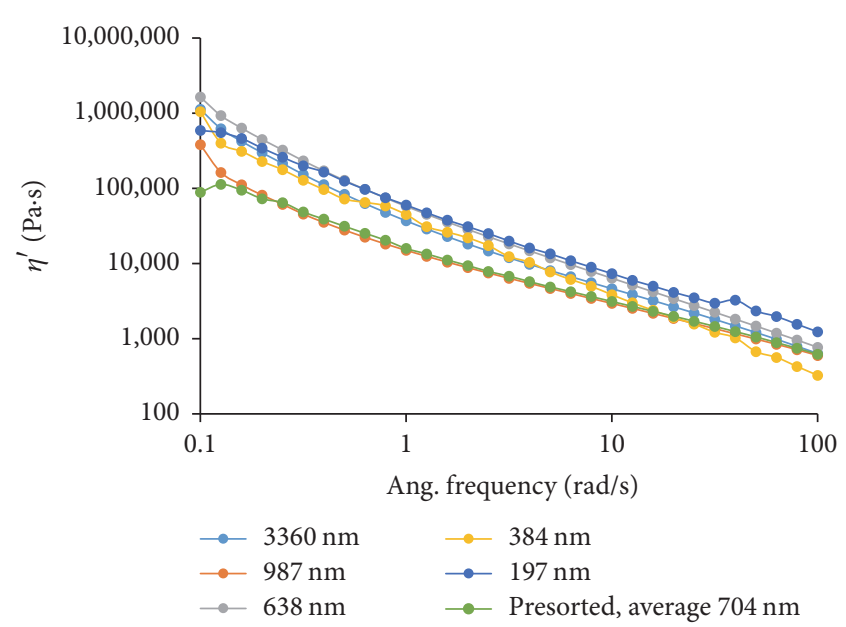

FIGURE 10: Dynamic viscosity of nanotube at various lengths ( $0.5 \mathrm{wt} . \%$ loading of SWNT, at data point $0.1 \mathrm{rad} / \mathrm{s}$ ).

$1.6 * 10^{5}<M^{n}<1.41 * 10^{7}$, radius of gyration can be written as

$$
R_{g}=1.37 * 10^{-2} * M^{0.595} \text {. }
$$

See [7].

In this work, the as-received PIB has a molecular weight $200,000 \mathrm{~g} / \mathrm{mol}$. Based on the equations above, its radius of gyration is $19.54 \mathrm{~nm}$. To measure the diameter of nanotube bundles, ImageJ Software was utilized to analyze the SEM pictures. The diameter at various positions was measured and compared with the scale bar for calculation. The obtained diameters range from 18.83 to $29.16 \mathrm{~nm}$. $R_{g}$ of PIB is not only comparable to this size, but also stays within this range. Thus, the perquisite for conformational change was completely satisfied. 
3.3. Differential Scanning Calorimetry. $T_{g}$ shift marks the change in polymer conformation, including extent of entanglement and packing density. More specifically, the shift towards to lower temperature refers to a loose arrangement of macromolecules. According to Table 2 , a $6-7^{\circ} \mathrm{C} T_{g}$ reduction occurs in the 0.02 and 0.05 wt.\% samples. As discussed above, nanotube bundles intertwined with macromolecules of PIB, reducing its entanglement. Meanwhile, the rigid-rod-like nanotube brings free volume itself, which further reduces the packing density of polymer. At low nanotube loadings, the combined effect of these two factors leads to a negative $T_{g}$ shift.

At higher loadings, difficulty arises in the isolation and dispersion of nanotubes in the PIB matrix. A reasonable assertion could be made that this is a regime within which there is a saturation of the number of nanotube bundles which have a size comparable with $R_{g}$ of the polymer. Beyond $5 \mathrm{wt} . \%$ nanoparticulate filler, the likely expression of rheological percolation dominates the structure-property relationships and we begin to see evidence of agglomerations, reducing any effects that would contribute to non-Einstein-like behavior.

It is well known that carbon nanotube can be a nucleation site that initiates polymer crystallization. The effect of nanotube length in this process, however, has hardly been studied. Li used polyethylene (PE) with a molecular weight $800 \mathrm{~g} / \mathrm{mol}$ [17]. PE is a highly crystalline polymer and can crystallize without nucleation agents. In Li's case, its molecular weight is also extremely low. Thus, the crystallization occurs readily when nanotubes are included. In our case, however, the PIB has a significantly large molecular weight at $200,000 \mathrm{~g} / \mathrm{mol}$, and it also has a methyl side group. Thus, crystallization could be more difficult. As discussed above, PIB is highly amorphous. Interestingly, the nanotubes of longer length fail to initiate crystallization, while short ones do. There could be multiple reasons for this difference. On one hand, the nanotubes have a large free volume, being highly rigid-rod-like and poorly deformable, and also the attachment of polymer is limited to steric hindrance and Van der Waals effects. Thus, the effect of free volume and the difficulty of interactions of the polymer could be increased with nanotube size and length. On the other hand, the formation of nanotube networks is easily facilitated and dense at longer lengths. The overlap and mingled arrangement of long length nanotube also provided poor surface area and space for polymer nucleation and lamellar growth.

Another interesting finding is that, among the various lengths of nanotube tested, the $638 \mathrm{~nm}$ length samples result in a larger degree of crystallization than the shorter ones. It seems that a minimum length of nanotube is required for optimum nucleation initiation. According to Binsbergen, the nucleation theory of Hoffman and Lauritzen also applies to polymer [25]. Nucleating agents should at least have a size comparable to the thickness of the lamella, to initiate heterogeneous nucleation of the polymer. Thus, the nanotube at an average length of $197 \mathrm{~nm}$ fails to achieve the full potential for nucleation. Furthermore, according to Table 1, the presorted, mixed length sample has the highest concentration of longer length SWNT comparatively, which stops crystallization as discussed before. Thus, although its average length is close to $638 \mathrm{~nm}$, a contrasting difference in crystallization behavior exists.

Finally, the reduced crystallinity degree at higher weight fractions, 0.25 and $0.5 \mathrm{wt} . \%$, is highly possibly due to an increasing of nanotube agglomeration and reduced concentration of nucleation sites of favorable size. However, the crystallization of PIB is not desirable for viscosity control, which will be discussed in the following section.

3.4. Rheological Testing. According to Table 4, the dynamic viscosity of PIB mixed with presorted nanotubes does not increase monotonously with weight fractions, which indicates the effect of conformational change and the occurrence of non-Einstein viscosity behavior. The 0.02 and $0.05 \mathrm{wt} . \%$ samples showed reduced viscosity compared to the pure PIB, for multiple reasons. On the one hand, the rigid-rodlike nanotube introduces free volume and decreases the packing density of PIB. On the other hand, it reduces the entanglement of polymer and further decreases viscosity. This conformational change was verified by $T_{g}$ testing recorded in Table 2, where the 0.02 and $0.05 \mathrm{wt} \%$ samples had a $T_{g}$ reduction as high as $6-7^{\circ} \mathrm{C}$. However, at high weight fractions of nanotube filler, the viscosity increases and finally becomes larger than that of PIB due to the saturated solubility of isolated nanotubes and the formation of large clustering. Thus, the weight fraction of nanotube should be carefully controlled for the purpose of viscosity modification, even in unsorted, unmodified SWNT additive, composites, and mixtures.

At a weight fraction of $0.1 \mathrm{wt} . \%$, the mixture with $638 \mathrm{~nm}$, $384 \mathrm{~nm}$, and $197 \mathrm{~nm}$ length nanotubes, as discussed above, showed significant crystallization. They also showed the highest viscosity due to the contribution of the crystalline region, which is still not completely melted at the testing temperature. Furthermore, the longer length nanotube samples still result in viscosity one order larger than the pristine presorted nanotube samples. Network structure is more easily formed in longer length nanotubes, which increases the viscosity. The longer length nanotubes therefore cause more resistance to rheological flow due to its rigid-rod-like morphology and their onset of network formations. Comparatively, as the weight fraction increases from 0.1 to $0.25 \mathrm{wt} . \%$, the dynamic viscosity of all samples shows an increase. In particular, the viscosity increases significantly for the longer length SWNT fractions of 3360 and $987 \mathrm{~nm}$, which can be attributed to lower critical percolation concentrations, for nanotube network formations, with higher frequency throughout the polymer matrix. Finally, crystallization was suppressed in the presorted samples that are rich in long length SWNTs. This could result from the shorter average length SWNTs present, compared to the 3360 and $987 \mathrm{~nm}$ samples, which would render reduced steric hindrance effects and shift the percolations concentration to higher values for network formation. The presorted, mixed length samples currently display the lowest viscosity profile of all the samples studied in this work.

\section{Materials and Methods}

4.1. Apparatus and Reagents. SWNTs were purchased from Southwest Nanotechnology, CoMoCAT type of synthesis, 
95\% purity. Sodium Deoxycholate Detergent (DOC) and Dichloromethane (DCM) were purchased from Fisher Scientific. The OptiPrep density gradient solution (60 wt $\%$ iodixanol) was purchased from Sigma Aldrich. A Misounic Ultrasound liquid processer, Qsonica LLC, was used for ultrasound treatment and mixing. A Sorvall Ultracentrifuge (Thermo Fisher) was used for density centrifugation. A PerkinElmer UV-Vis-NIR spectrometer was utilized to study the length of each layer after separation. Scanning Electron Microscopy (SEM, Quanta FEG 650) was used to observe morphology of length sorted nanotube samples. An AFM (ezAFM, NANOMAGNETIC Instruments) was utilized to study the topography and amplitude of nanotube bundles. A DSC Q 100 (TA Instruments) was used to study the glass transition and crystalline behavior of nanotube polymer mixture. An Ares 2000 Rheometer (TA Instruments) was used to measure the dynamic viscosity. Finally, Fourier transform infrared spectroscopy (FTIR, NICOLET 4700 FTIR, Thermo Electron Corporation) was used to further study the crystallinity degree quantitatively.

4.2. Length Separation of SWNT. $900 \mathrm{mg}$ of SWNT was blended with $900 \mathrm{ml} 2$ wt.\% DOC surfactant solution. The as-formed $1 \mathrm{mg} / \mathrm{ml}$ nanotube solution was treated with tip ultrasound mixing at amplitude 40 , power $14 \mathrm{~W}$, and $1 \mathrm{~h}$ in an ice bath. The solution was then directly treated with ultracentrifugation at $5^{\circ} \mathrm{C}, 30 \mathrm{mins}$ at a rotation speed $20300 \mathrm{rpm}$. This step removes large bundles formed during ultrasound treatment, rendering the presorted nanotube fractions. Then the density gradient was made by mixing iodixanol solution and deionized water in various ratios. The $13 \mathrm{ml}$ centrifuge tube was used, and from top to bottom the composition of density gradient was $6 \mathrm{ml}-18 \%, 4 \mathrm{ml}-25 \%$, and $2 \mathrm{ml}-30 \%$ iodixanol solution. The bottom solution was made by mixing OptiPrep with SWNT aqueous solution directly, instead of DI water. Then the SWNT-density gradient solution was treated with ultracentrifugation at $5^{\circ} \mathrm{C}$, at $39600 \mathrm{rpm}$, and for $12 \mathrm{hrs}$. The final product was separated by hand pipetting removing $0.75 \mathrm{ml}$ per layer.

4.3. Fabrication of SWNT-PIB Nanocomposites. $500 \mathrm{mg}$ of virgin high MW PIB was weighted and mixed with $30 \mathrm{ml}$ of Dichloromethane. Ultrasound treatment was applied to the mixture at an amplitude 70, at power $14 \mathrm{~W}$, and for $2 \mathrm{hrs}$. The nanotube was mixed with PIB at varying weight fractions and lengths separately. First, aqueous solution of presorted nanotube was dropped into the mixture at various weight fractions including $0.05,0.1,0.25,0.5,1$, and $5 \mathrm{wt} . \%$. Then ultrasound treatment was applied for another one hour at the same amplitude. The final mixture was dried vacuum oven overnight. The same treatment was repeated to as-received PIB as the control group. Later PIB was further mixed with nanotubes after length sorting. The selected lengths include $3360 \mathrm{~nm}, 987 \mathrm{~nm}, 638 \mathrm{~nm}, 384 \mathrm{~nm}$, and $197 \mathrm{~nm}$. They were mixed with PIB using the same technique and posttreatment, but all at a weight fraction of $0.1 \mathrm{wt} . \%$. Two repeated groups of test were conducted to nanotube of the same five lengths, but at weight fraction 0.25 and 0.5 wt. $\%$.
4.4. Characterizations. UV-Vis-NIR spectroscopy was utilized to study the length of each layer after separation. The test used wavelength from 400 to $1100 \mathrm{~nm}$. The curve was normalized at $775 \mathrm{~nm}$ where amplitude was reset to 1 . Later the absorbance at $984 \mathrm{~nm}$ was recorded. The equation below was used to calculate the final length.

$$
L(\mathrm{~nm})=\left(\frac{A 984 \mathrm{~nm}}{A 775 \mathrm{~nm}}-1\right) * 160.4 \mathrm{~nm} .
$$

See [21].

In order to determine the concentration of SWNT aqueous solution, $50 \mathrm{ml}$ of nanotube solution was tested by UVVis-NIR spectroscopy, and the absorbance at $775 \mathrm{~nm}$ was recorded. Then it was deposited on the filter paper by vacuum filtration. The weight difference of filter before and after inclusion of nanotube was recorded as the overall weight of SWNT. Since the concentration of CoMoCAT SWNT is directly proportional to the absorbance at $775 \mathrm{~nm}$, the concentration of any other nanotube solution would be calculated using the standard sample measured above.

Scanning Electron Microscopy (SEM) was performed at a Secondary Electron (SE) mode with spot size 4.0 and accelerating voltage $20 \mathrm{kV}$. The magnification ranges from 50 to $100 \mathrm{k}$ times. The ImageJ Software was applied to estimate the diameter of nanotube bundles with reference to scale bar. An AR 2000 Rheometer was utilized for viscosity measurement. The frequency sweep was conducted to all samples at $160^{\circ} \mathrm{C}$, from 0.1 to $100 \mathrm{rad} / \mathrm{s}$. The constant parameter was set as $0.5 \%$ strain. Five sweeps at each frequency were automatically conducted by the rheometer and averaged as the final data point. The thermal properties were studied by Differential Scanning Calorimetry (DSC) Q 100, under indium and zinc calibration standard. Uniformly weighted samples of 5-20 mg were sealed within perforated aluminum pans and went through a temperature ramp mode from -80 to $440^{\circ} \mathrm{C}$. The ramp rate was $5^{\circ} \mathrm{C} / \mathrm{min}$ under a nitrogen atmosphere.

\section{Conclusions}

In this work, the length separation of SWNTs was conducted and analyzed. The effect of nanotube length and weight fraction on PIB was studied. The result shows that nanotube facilitates conformational changes to the polymer and causes non-Einstein-like viscosity reduction at lower weight fractions. The shorter length nanotubes of $638 \mathrm{~nm}$ proved to be ideal nucleation agents to initiate crystallization of PIB, while longer length nanotubes do not show the same initiation responses. The longer length nanotubes form networks easily and dramatically increase viscosity. We believe this work will broaden our understanding of SWNT-polymer nanocomposites, particularly in the control of nanotube length and weight fractions to achieve optimum properties.

\section{Conflicts of Interest}

The authors declare no conflicts of interest. 


\section{Authors' Contributions}

Daneesh O. Simien is the laboratory's principal investigator. Daneesh O. Simien, Clayton E. Simien, and Hanxiao Huang conceived and designed the experiments. Hanxiao Huang performed the experiments and analyzed the data. Bohao Li conducted research in length separation of carbon nanotubes.

\section{Acknowledgments}

The authors acknowledge financial support from the UAB faculty development grant program (FDGP). They also sincerely appreciate operation and instruction from William Monroe, SEM technician in the UAB School of Engineering.

\section{References}

[1] M. Endo, S. Iijima, and M. S. Dresselhaus, Carbon Nanotubes, Elsevier, 1997.

[2] K. Song, Y. Zhang, J. Meng et al., "Structural polymer-based carbon nanotube composite fibers: understanding the processingstructure-performance relationship," Materials, vol. 6, no. 6, pp. 2543-2577, 2013.

[3] P. R. Bandaru, "Electrical properties and applications of carbon nanotube structures," Journal of Nanoscience and Nanotechnology, vol. 7, no. 4-5, pp. 1239-1267, 2007.

[4] A. Moisala, Q. Li, I. A. Kinloch, and A. H. Windle, "Thermal and electrical conductivity of single- and multi-walled carbon nanotube-epoxy composites," Composites Science and Technology, vol. 66, no. 10, pp. 1285-1288, 2006.

[5] M. E. Mackay, T. T. Dao, A. Tuteja et al., "Nanoscale effects leading to non-einstein-like decrease in viscosity," Nature Materials, vol. 2, no. 11, pp. 762-766, 2003.

[6] M. E. Mackay, A. Tuteja, P. M. Duxbury et al., "General strategies for nanoparticle dispersion," Science, vol. 311, no. 5768, pp. 17401743, 2006.

[7] L. J. Fetters, N. Hadjichristidis, J. S. Lindner, and J. W. Mays, "Molecular weight dependence of hydrodynamic and thermodynamic properties for well-defined linear polymers in solution," Journal of Physical and Chemical Reference Data, vol. 23, no. 4, pp. 619-640, 1994.

[8] S. Jain, J. G. P. Goossens, G. W. M. Peters, M. Van Duin, and P. J. Lemstra, "Strong decrease in viscosity of nanoparticle-filled polymer melts through selective adsorption," Soft Matter, vol. 4, no. 9, pp. 1848-1854, 2008.

[9] X.-L. Xie, Q.-X. Liu, R. K.-Y. Li et al., "Rheological and mechanical properties of $\mathrm{PVC} / \mathrm{CaCO}_{3}$ nanocomposites prepared by in situ polymerization," Polymer, vol. 45, no. 19, pp. 6665-6673, 2004.

[10] J. W. Cho and D. R. Paul, "Nylon 6 nanocomposites by melt compounding," Polymer, vol. 42, no. 3, pp. 1083-1094, 2001.

[11] C. Roberts, T. Cosgrove, R. G. Schmidt, and G. V. Gordon, "Diffusion of poly(dimethylsiloxane) mixtures with silicate nanoparticles," Macromolecules, vol. 34, no. 3, pp. 538-543, 2001.

[12] A. Tuteja, M. E. Mackay, C. J. Hawker, and B. Van Horn, "Effect of ideal, organic nanoparticles on the flow properties of linear polymers: non-einstein-like behavior," Macromolecules, vol. 38, no. 19, pp. 8000-8011, 2005.

[13] A. Tuteja, P. M. Duxbury, and M. E. Mackay, "Multifunctional nanocomposites with reduced viscosity," Macromolecules, vol. 40, no. 26, pp. 9427-9434, 2007.
[14] R. G. Schmidt, G. V. Gordon, C. A. Dreiss et al., "A critical size ratio for viscosity reduction in poly(dimethylsiloxane)polysilicate nanocomposites," Macromolecules, vol. 43, no. 23, pp. 10143-10151, 2010.

[15] A. Petriccione, M. Zarrelli, V. Antonucci, and M. Giordano, "Aggregates of chemically functionalized multiwalled carbon nanotubes as viscosity reducers," Materials, vol. 7, no. 4, pp. 3251-3261, 2014.

[16] Q. Zhang, D. R. Lippits, and S. Rastogi, "Dispersion and rheological aspects of SWNTs in ultrahigh molecular weight polyethylene," Macromolecules, vol. 39, no. 2, pp. 658-666, 2006.

[17] L. Li, W. Wang, E. D. Laird, C. Y. Li, M. Defaux, and D. A. Ivanov, "Polyethylene/carbon nanotube nano hybrid shishkebab obtained by solvent evaporation and thin-film crystallization," Polymer, vol. 52, no. 16, pp. 3633-3638, 2011.

[18] L. Tan, Y. Chen, W. Zhou, S. Ye, and J. Wei, "Novel approach toward poly(butylene succinate)/single-walled carbon nanotubes nanocomposites with interfacial-induced crystallization behaviors and mechanical strength," Polymer, vol. 52, no. 16, pp. 3587-3596, 2011.

[19] M. L. Minus, H. G. Chae, and S. Kumar, "Single wall carbon nanotube templated oriented crystallization of poly(vinyl alcohol)," Polymer, vol. 47, no. 11, pp. 3705-3710, 2006.

[20] S.-N. Li, Z.-M. Li, M.-B. Yang, Z.-Q. Hu, X.-B. Xu, and R. Huang, "Carbon nanotubes induced nonisothermal crystallization of ethylene-vinyl acetate copolymer," Materials Letters, vol. 58, no. 30, pp. 3967-3970, 2004.

[21] J. A. Fagan, M. L. Becher, J. Chun, and E. K. Hobbie, "Length fractionation of carbon nanotubes using centrifugation," Advanced Materials, vol. 20, no. 9, pp. 1609-1613, 2008.

[22] D. R. Paul and L. M. Robeson, "Polymer nanotechnology: nanocomposites," Polymer, vol. 49, no. 15, pp. 3187-3204, 2008.

[23] D. Simien, J. A. Fagan, W. Luo, J. F. Douglas, K. Migler, and J. Obrzut, "Influence of nanotube length on the optical and conductivity properties of thin single-wall carbon nanotube networks," ACS Nano, vol. 2, no. 9, pp. 1879-1884, 2008.

[24] J. A. Fagan, M. Zheng, V. Rastogi et al., "Analyzing surfactant structures on length and chirality resolved $(6,5)$ single-wall carbon nanotubes by analytical ultracentrifugation," ACS Nano, vol. 7, no. 4, pp. 3373-3387, 2013.

[25] F. L. Binsbergen, "Heterogeneous nucleation in the crystallization of polyolefins. III. Theory and mechansim," Journal of Polymer Science Part A-2: Polymer Physics, vol. 11, no. 1, pp. 117135, 1973. 

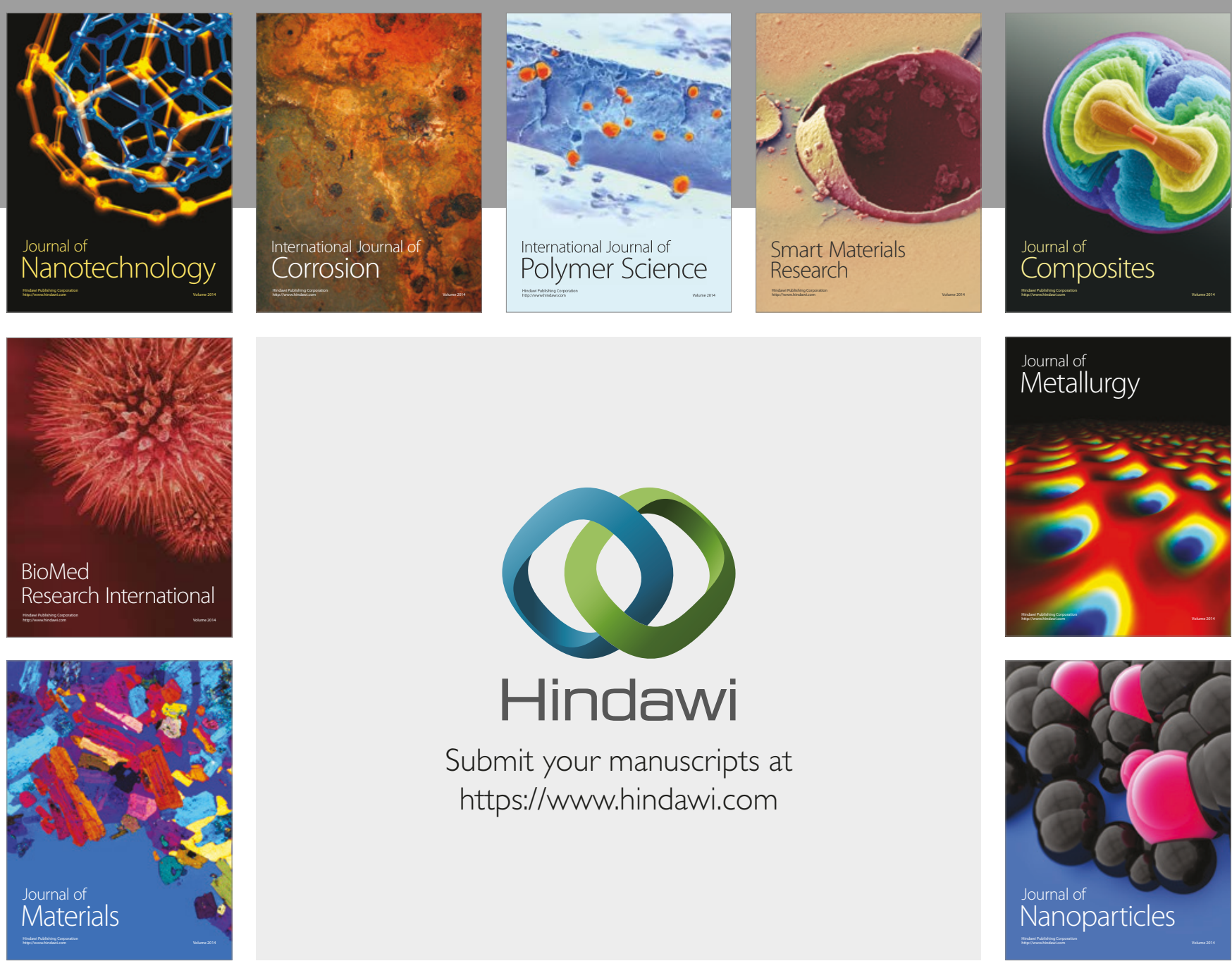

\section{Hindawi}

Submit your manuscripts at

https://www.hindawi.com
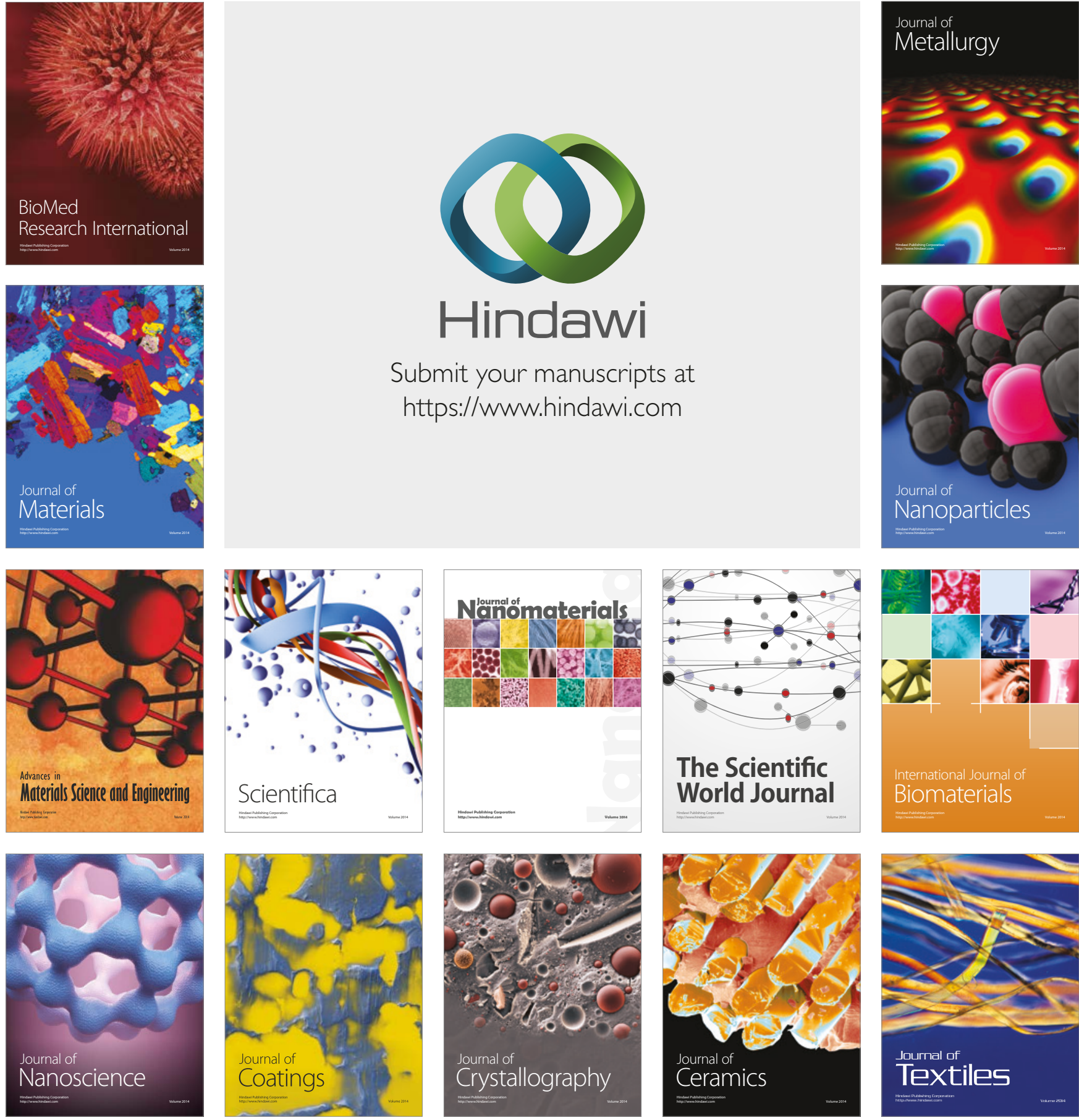

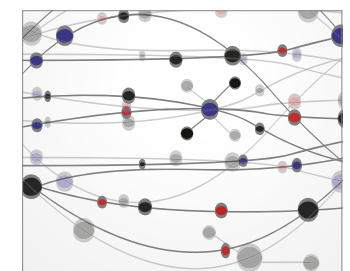

The Scientific World Journal
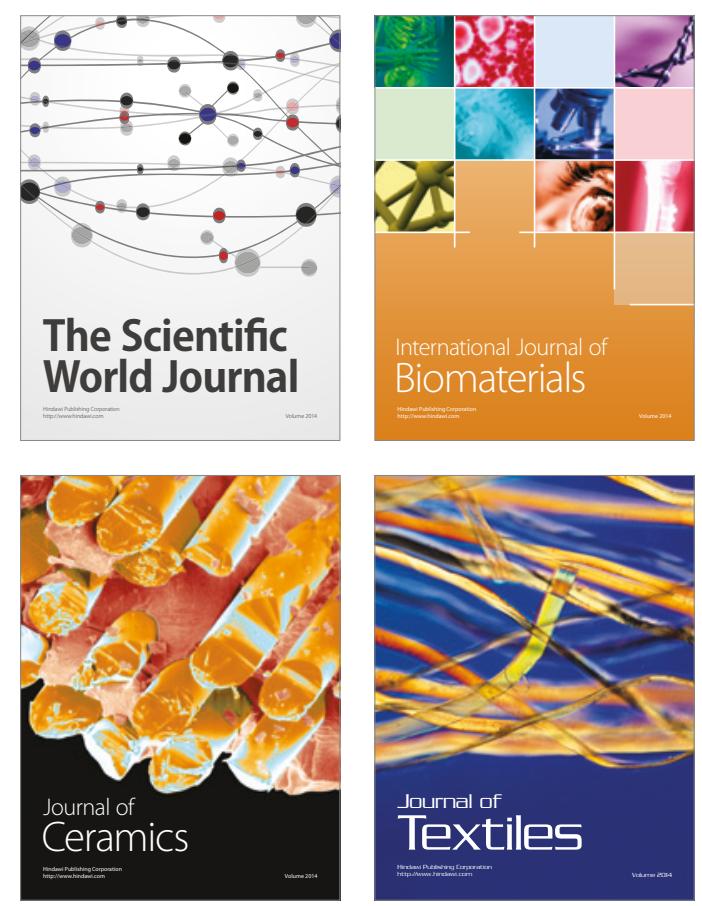